Research Article

\title{
Ozone Oxidation of Kraft Bamboo Pulp for Preparation of Nanofibrillated Cellulose
}

\author{
Mingyou Liu, ${ }^{1}$ Xia Chen, ${ }^{1}$ and Xiaofei Tian $\mathbb{D D}^{2,3}$ \\ ${ }^{1}$ School of Light Industry, South China University of Technology, Guangzhou 510006, China \\ ${ }^{2}$ School of Biology and Biological Engineering, South China University of Technology, Guangzhou 510006, China \\ ${ }^{3}$ Guangxi Key Laboratory of Clean Pulp \& Papermaking and Pollution Control, College of Light Industry and Food Engineering, \\ Guangxi University, Nanning 530004, China
}

Correspondence should be addressed to Xiaofei Tian; xtien@scut.edu.cn

Received 9 March 2018; Revised 16 May 2018; Accepted 21 May 2018; Published 3 July 2018

Academic Editor: Marta Fernández-García

Copyright (c) 2018 Mingyou Liu et al. This is an open access article distributed under the Creative Commons Attribution License, which permits unrestricted use, distribution, and reproduction in any medium, provided the original work is properly cited.

The influence of ozonation on the homogenization of Kraft bamboo pulp (KBP) for the production of nanofibrillated cellulose (NFC) was studied. Using optimized conditions for ozonation, that is, pulp consistency 35\%, ozone dosage $0.87 \%(v / w)$, and $\mathrm{pH}=2.5$, the kappa number and viscosity of $\mathrm{KBP}$ decreased from 10.8 to 2.8 and from $1024 \mathrm{~mL} \cdot \mathrm{g}^{-1}$ to $258 \mathrm{~mL} \cdot \mathrm{g}^{-1}$, respectively, while the crystallinity and carboxylate content increased from $36.2 \%$ to $48 \%$ and $0.93 \mathrm{mmol} / \mathrm{g}$ to $1.26 \mathrm{mmol} / \mathrm{g}$, respectively. The ozonation-treated $\mathrm{KPB}$ was used as the substrate for NFC preparation through homogenization. With a width of $10-20 \mathrm{~nm}$ and length $\geq 250 \mathrm{~nm}$, the prepared NFC had a high aspect ratio of length versus width. Ozonation can be used as an alternative approach to promote the efficient production of NFC from KBP.

\section{Introduction}

Cellulose is a natural, renewable material commonly used in the production of biocompatible or environmentally friendly product $[1,2]$. It is also used as a low-cost filler for sustainable composite materials and, therefore, could be applied as a reinforcing element in polymer matrices [3]. In particular, bioderived, nanosized cellulose nanofillers have attracted great attention because they have a stronger reinforcing potential than individual cellulose fibers [4]. Nanofibrillated cellulose (NFC) is a common nanosized material for which at least one dimension is at the nanometer level. The diameters of NFC particles are usually below $100 \mathrm{~nm}$. Furthermore, the high strength, high Young's modulus, low coefficient of thermal expansion, and transparency of NFC make it one of the most promising reinforcing elements to be used in a range of products.

NFC can be mechanically separated from woody biomass $[5,6]$. The natural herbal fibers have a complex multilayer structure, which contributes to a complex aggregation of fibers. Moreover, there is a large extent of hydrogen bonding among the cellulose microfibrils. The natural cellulose material showed a strong resistance against mechanical fibrillation. Therefore, the mechanical preparation of NFC is a high-energy process that requires special equipment, which is not conducive to industrial production [7].

To improve the production efficiency of NFC, the raw materials are pretreated prior to mechanical shearing. Chemical pretreatment methods, including oxidation, acid hydrolysis, or organic solvent dissolution, are commonly applied to remove lignin and hemicellulose, to reduce the crystallinity of the cellulose, and to promote the hydrophilicity of the material. Besbes et al. [8] reported a TEMPO(2,2,6,6-tetramethylpiperidine-1-oxyl radical-) mediated oxidation procedure, which assisted the defibrillation process by reducing the number of required passes and preventing the cellulose fibers from clogging in the homogenizer $[9,10]$. Acid pretreatment is typically performed in a heated acidic solution ( $64 \%$ concentrated sulfuric acid) with ultrasound or mechanical stirring in order to hydrolyze 
the cellulose fibers. Unfortunately, most chemical methods suffer from harsh reaction conditions and result in environmental pollution.

Ozone is a green oxidizing reagent that has been important in pulp bleaching because of its strong capacity for lignin removal. In addition, it could also efficiently perform ozonation (ozone oxidization) on the carbohydrate polymers in the fiber. While ozone is a strong oxidant, ozonation of cellulose leads to a minor oxidation reaction mostly at the C-6 position $[11,12]$. The reaction could increase the number of negatively charged species on the fiber surface, similar to the TEMPO-mediated oxidation system, to promote fibrillation during the homogenization process. The ozonation could spur the development of new applications and markets for CNF with the advantages of low energy and low chemical requirement [13].

Bleached pulp from the pulping and paper industries has been extensively used as a raw material for the preparation of NFC $[14,15]$. Although they are readily available and contain high-purity cellulose, bleached pulp and pure cellulose fibers are more expensive than unbleached pulp, especially when considering large-scale production. However, unbleached pulp has a high lignin content that should be removed from the fibers as much as possible before use. The retained fiber should also be broken down and turned into filaments before homogenization to avoid clogging the high-pressure homogenizer.

Considering the high efficiencies of delignification as well as good oxidizing selectivity, direct application of the ozonation method on the pretreatment of the unbleached Kraft pulp instead of pure cellulose fibers for the NFC preparation would be feasible. In this study, the effect of ozonation on the physical properties of Kraft bamboo pulp (KPB) for subsequent mechanical production of NFC was determined. The objective of this work is to demonstrate that the ozone oxidation could be applied in conjunction with mechanical homogenization for produce CNF from the Kraft pulps.

\section{Experiments}

2.1. Materials. The KBP sample was obtained from a local pulp mill (Sichuan, China). The kappa number, viscosity, and water consistency of the KBP were $10.1,1024 \mathrm{~mL} \cdot \mathrm{g}^{-1}$, and $33 \%$, respectively. Before use, the KBP was activated via oxidation with oxygen at $100^{\circ} \mathrm{C}$ for $100 \mathrm{~min}$. Then, $60 \mathrm{~g}$ (dry weight (DW)) of EKP was sealed in a $2 \mathrm{~L}$ reactor (Dawn Precision Instrument Co., Ltd, Shandong, China) with $700 \mathrm{~mL}$ of a solution containing $10 \%(w / v) \mathrm{NaOH}$, $1 \%(w / v) \mathrm{MgSO}_{4}$, and $30 \%(v / v) \mathrm{H}_{2} \mathrm{O}_{2}$. The pressure in the reactor was $0.6 \mathrm{Mpa}$ at $100^{\circ} \mathrm{C}$.

\subsection{NFC Preparation}

2.2.1. PFI Refining. The KBP was adjusted to a $10 \%(w / w)$ oven-dried (OD) consistency with distilled water and dispersed evenly. According to QB/T 1463-2010, the pulp was loaded and mechanically sheared on a PFI refiner (Mark VI, Hamjern Maskin A/S, Hamar, Norway) for 1000 revolutions.
TABLE 1: Reaction conditions for the ozonation stage.

\begin{tabular}{lcccc}
\hline Condition & $A$ & $Z$ & $E$ & $P$ \\
\hline Pulp consistency $(w / w)$ & 6.0 & $25-45$ & 7.0 & 10 \\
Temperature $\left({ }^{\circ} \mathrm{C}\right)$ & 60 & $\mathrm{RT}$ & 70 & 90 \\
Time $(\mathrm{min})$ & 60 & $4-12$ & 60 & 100 \\
$\mathrm{pH}$ & $\mathrm{NA}$ & $1.5-3.5$ & $\mathrm{NA}$ & $\mathrm{NA}$ \\
$\mathrm{H}_{2} \mathrm{SO}_{4}(w / w)$ & 2.0 & - & - & - \\
$\mathrm{O}_{3}(v / w)$ & - & $0.4-1.2$ & - & - \\
$\mathrm{NaOH}(w / w)$ & - & - & 1.5 & 0.5 \\
$\mathrm{H}_{2} \mathrm{O}_{2}(w / w)$ & - & - & - & 2.5 \\
\hline
\end{tabular}

RT: room temperature $\left(25^{\circ} \mathrm{C}\right)$; NA: not available.

2.2.2. Ozonation. The ozonation process includes 4 stages: pretreatment with sulfuric acid $(A)$, ozone oxidation $(Z)$, alkali treatment with sodium hydroxide $(E)$, and hydrogen peroxide treatment $(P)$. Table 1 shows the reaction conditions applied in each of the 4 stages.

In the $A, E$, and $P$ stages, the BKP was sealed in polyethylene bags and incubated at the designated temperature in a water bath. During the reaction, the BKP sample was mixed every $20 \mathrm{~min}$ by hand kneading the bags. After each stage of the reaction, the collected BKP samples were washed thoroughly with distilled water (5 times $w / v)$ until the $\mathrm{pH}$ reached 7 .

Before ozone oxidation, the washed pulp was dewatered and sealed in polyethylene bags for $12 \mathrm{~h}$ at room temperature to maintain consistent water content. Then, the sample was placed in a self-designed rotary reactor [16]. Ozone with a flow of $120 \mathrm{~L} / \mathrm{Nm}^{3}$ was pumped into a $2.5 \mathrm{~L}$ bleaching reactor through Teflon tubes at a flow rate of $800 \mathrm{~mL} / \mathrm{min}$. The ozone was produced by an ozone generator (KCF-SF100B, Koner, Jiangsu, China) using the compressed pure $\mathrm{O}_{2}$ as the gas supply. The reactor was rotating at $60 \mathrm{r} / \mathrm{min}$ at $25^{\circ} \mathrm{C}$.

2.2.3. Mechanical Homogenization. The disintegration of ozone-treated KBP pulp was performed using a highpressure microfluidizer (Lab-scale, Nano DeBEE, USA) equipped with D5 and D10 nozzles. The KBP was dispersed in distilled water and diluted to a $0.1 \%(w / w)$ suspension. A $50 \mathrm{~mL}$ suspension was continuously homogenized for 3 times at 10,000 psi and then twice at 25,000 psi.

\subsection{Characterization}

2.3.1. Kappa Number and Viscosity. The kappa number and viscosity of the BKP were determined according to GB/T 1546-1989 and GB/T 1548-2016, respectively. The BKP sample that was collected after ozonation was recognized as the treated BKP.

2.3.2. Determination of the Degree of Crystallinity. The X-ray diffraction (XRD) patterns of the original and treated BKP were recorded using a Bruker X-ray diffractometer (D8 Advance, USA) equipped with $\mathrm{Cu}-\mathrm{K} \alpha$ radiation $(\lambda=0.154$ $\mathrm{nm}$ ). The $2 \theta$ values were measured from $5^{\circ}$ to $40^{\circ}$ with a step 


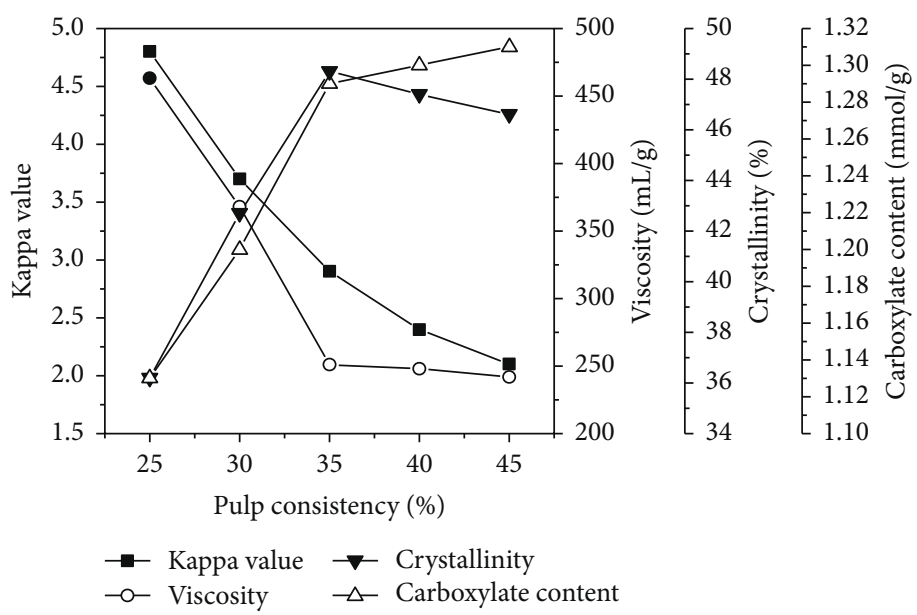

FIGURE 1: Effect of pulp consistency on kappa number, viscosity, crystallinity, and carboxylate content.

of $0.04^{\circ}$ and $0.2 \mathrm{~s}$. The crystallinity index (CrI) was calculated by formula [17] as follows:

$$
\mathrm{Cr} \%=\left[\frac{I_{002}-I_{\mathrm{am}}}{I_{002}}\right] \times 100
$$

where $I_{002}$ and $I_{\mathrm{am}}$ represent the intensity of the peaks that correlate to the 020 and $10 \overline{1}$ lattice planes of cellulose $I$ at 2 $\theta=22.5^{\circ}$ and $16.5^{\circ}$, respectively.

2.3.3. Carboxylate Content. The carboxylate content in BKP was determined using the conductometric-titration method. A $55 \mathrm{~mL}$ BKP suspension $(0.55 \%, w / w)$ was prepared in deionized water. After adding $5 \mathrm{~mL}$ of $0.01 \mathrm{M} \mathrm{NaCl}$, the $\mathrm{pH}$ was adjusted to 2.5 using $0.1 \mathrm{M} \mathrm{HCl}$. The mixture was stirred well and titrated with $0.1 \mathrm{M} \mathrm{NaOH}$. The total amount of carboxyl groups was calculated according to

$$
C=\frac{\left(V_{2}-V_{1}\right) \times C_{\mathrm{NaOH}}}{m},
$$

where $C(\mathrm{mmol} / \mathrm{g})$ is the carboxylate content, $V_{2}(\mathrm{~mL})$ is the added volume of the $\mathrm{NaOH}$ solution when the titration curves reached their peak point, $V_{1}(\mathrm{~mL})$ is the volume of $\mathrm{HCl}$ added to the mixture, $C_{\mathrm{NaOH}}(\mathrm{M})$ is the exact concentration of the $\mathrm{NaOH}$ solution, and $m$ is the weight of the dried product (g).

2.3.4. Electron Microscopy. The surface of the treated BKP and the nanostructure of the NFC product were observed with a Carl Zeiss Evo 18 scanning electron microscope (SEM; Germany) and H-7650 HITACHI transmission electron microscope (TEM; Japan), respectively. Before observation, the treated BKP was freeze-dried and coated with a gold film. The NFC product was transferred into a $2 \mathrm{~mL}$ tube and centrifuged at $8000 \mathrm{r} / \mathrm{min}$ for $5 \mathrm{~min}$ using a centrifuge (Eppendorf Vertrieb GmbH, Germany). The liquid supernatant was diluted 50 times with distilled water, and $20 \mu \mathrm{L}$ of the diluted supernatant was dropped on a copper sample disc and air dried. The SEM and TEM images were collected at an acceleration voltage of $5-15 \mathrm{kV}$ and $150 \mathrm{kV}$, respectively.
TABLE 2: The relationship between the ozone dosage and treatment time.

\begin{tabular}{lc}
\hline Ozone dosage (\%) & Time $(\mathrm{min})$ \\
\hline 0.42 & 4 \\
0.64 & 6 \\
0.87 & 8 \\
1.06 & 10 \\
1.21 & 12 \\
\hline
\end{tabular}

\section{Results and Discussion}

3.1. Ozonation of KBP. The selective oxidization of the hydroxymethyl groups on cellulose using a TEMPOmediated system led to significant improvements in the efficiency of mechanical processing in the preparation of individual NFC suspensions [18-20], which suggests that a net increase in the negative surface charge of the nanofibers could facilitate mechanical peeling of the fibers. Ozone is a strong oxidant that could simultaneously react with the residual lignin and oxidize the groups, such as aromatic rings and $-\mathrm{C}=\mathrm{C}$ - in the side chains, to improve the surface hydrophilicity of the fibers. In theory, ozone is a promising green reagent for pretreating unbleached pulp before the preparation of NFC; nevertheless, free radicals generated during ozonation could cause the degradation of the cellulose and carbohydrates. This potential problem can be combatted by controlling the intensity of the reaction.

In ozonation, pulp consistency, ozone consumption, and initial $\mathrm{pH}$ were considered as the key factors for an efficient oxidation process [21]. Their optimal levels were determined by single-factor experiments using the change in the KBP properties, that is, crystallinity, viscosity, kappa number, and carboxylate content, as the response factors.

3.1.1. Effect of Pulp Consistency. For the ozonation of highconsistency pulp, the optimal concentration of the $\mathrm{pH}$ and ozone dosage were $2-3$ and $0.4-1.2 \%$, respectively [22]. When the pulp consistency varied from $25 \%$ to $45 \%$, the 


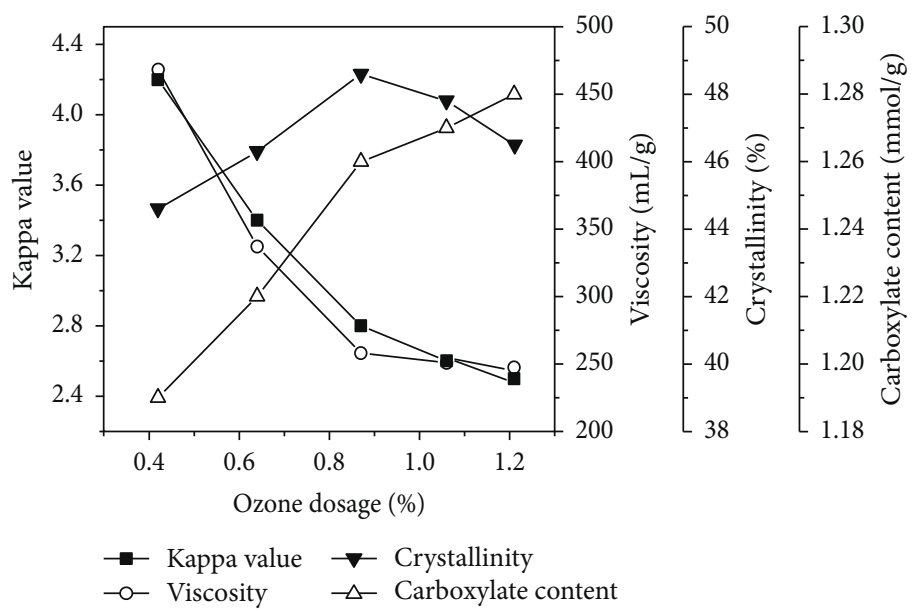

FIGURE 2: Effect of ozone dosage on kappa number, viscosity, crystallinity, and carboxylate content.

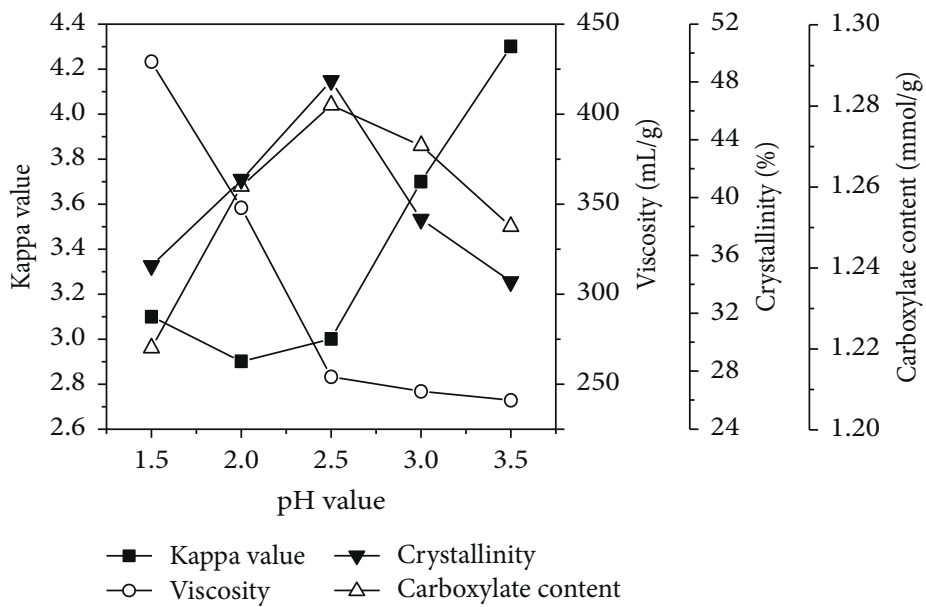

Figure 3: Effect of pH on kappa number, viscosity, crystallinity, and carboxylate content of the pulp.

TABLE 3: Change in the properties of the KBP.

\begin{tabular}{lcc}
\hline & Original KBP & $\begin{array}{c}\text { Treated KBP } \\
\text { after ozonation }\end{array}$ \\
\hline Kappa number & 10.8 & 2.8 \\
Viscosity $\left(\mathrm{mL} \cdot \mathrm{g}^{-1}\right)$ & 1024 & 258 \\
Crystallinity degree $(\%)$ & 36.2 & 48 \\
Carboxylate content $(\mathrm{mmol} / \mathrm{g})$ & 0.93 & 1.26 \\
\hline
\end{tabular}

acquired experimental $\mathrm{pH}$ and ozone dosage were 2.5 and $0.87 \%$, respectively.

Figure 1 shows the change in kappa number, viscosity, crystallinity, and carboxylate content for KBP when different pulp consistencies are used in the ozonation process. When the pulp consistency increased from $25 \%$ to $35 \%$, the kappa number and viscosity decreased rapidly. This indicated accelerated delignification and degradation of the cellulose. Ozonation is recognized as a complex mass-transfer process including stages of both solid chemical absorption and gasliquid mass transfer [23, 24]. Because ozone is nearly insoluble in water, the resistance of mass transfer was supposed to be generally dependent on the water film between the ozone and the surface of the BKP. With accelerated ozonation, the increased pulp consistency may have led to a thinner water film on the BKP. This is consistent with the measured increase in crystallinity and carboxylate content that was caused by the oxidization of the amorphous cellulose and hydroxyl groups, respectively. When the pulp consistency exceeded 35\%, the rates of decrease in the Kappa number and viscosity, as well as the rate of increase in the carboxylate content, were slowed down. However, a decrease in crystallinity was observed. This indicated that the cellulose had an intense reaction with the ozone.

Based on these results, the critical level of pulp consistency for the ozonation reaction was 35\%, and a 35\% pulp consistency was applied to subsequent experiments.

3.1.2. Effect of Ozone Dosage. At a constant ozone composition $\left(120 \mathrm{~L} / \mathrm{Nm}^{3}\right)$ and gas flow rate $(800 \mathrm{~mL} / \mathrm{min})$, the ozone dosage is mostly dependent on the amount of time the oxidation reaction is continued. In this experiment, 


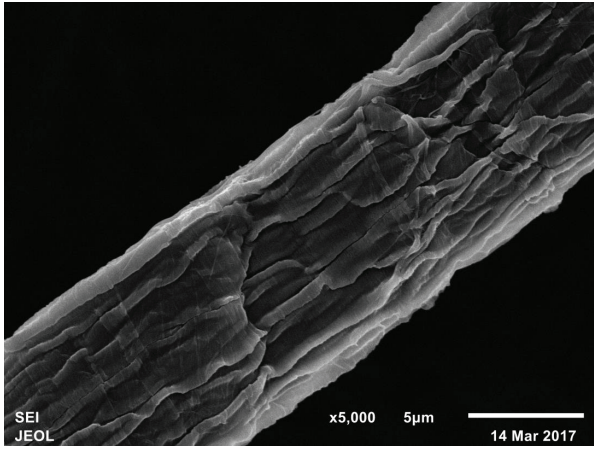

(a)

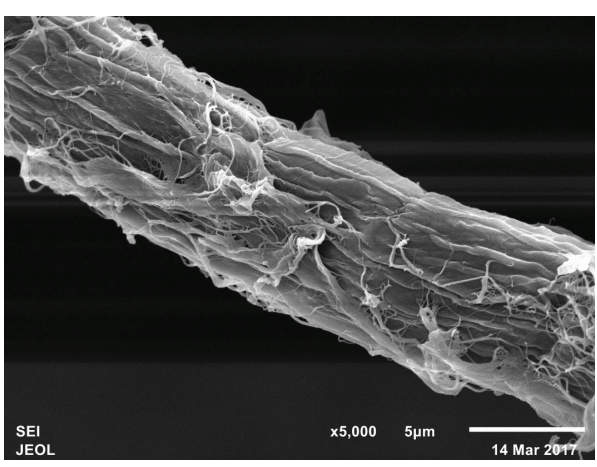

(b)

FIGURE 4: The morphology of the BKP fiber and the prepared BKP fiber before (a) and after (b) ozonation.

different ozone dosages were applied in the ozonation process by controlling the reaction time (Table 2). The effect of the ozone dosage on the BKP properties was examined at $\mathrm{pH}=2.5$ and $35 \%$ pulp consistency.

As the ozone dosage increased, the kappa number and viscosity gradually decreased. The rate of decline decreased when the ozone dosage exceeded $0.87 \%$ (Figure 2). Conversely, the crystallinity and carboxylate content increased notably when the ozone dosage increased from $0.42 \%$ to $0.87 \%$. When the ozone dosage was over $0.87 \%$, the crystallinity started to decrease.

The reduction in the viscosity indicated the degradation of cellulose. This was correlated to the ozone-induced delignification reaction, which could generate a large number of free radicals. These free radicals could attack the chemical bonds within the carbohydrates and cause the degradation of cellulose in the BKP. Because the cellulose crystal structure is also impacted by the free radical attacks, the crystallinity of the cellulose decreased as the oxidation time increased. The increasing carboxylate content with increasing ozone dosage indicated the oxidation of hydroxyl groups to carboxyl groups by ozonation.

3.1.3. Effect of $p H$. The rate of ozone self-decomposition could be reduced by the proper $\mathrm{H}^{+}$concentration within the pulp. The effect of $\mathrm{pH}$ on the properties of the pulp was studied using a $35 \%$ pulp consistency and ozone dosage of $0.87 \%$.

Figure 3 shows that the lowest kappa number and the highest crystallinity and carboxylate content were achieved when $\mathrm{pH}=2.5$. When $\mathrm{pH}<2.5$, the ozone decomposed less and the delignification rate increased, while the cellulose decomposition decreased. Higher $\mathrm{pH}$ led to lower viscosity but a higher kappa number, as intensive decomposition of the ozone could generate a large amount of hydroxyl radicals to oxidize the cellulose polymers. The overconsumption of ozone to produce hydroxyl radicals had a negative effect on delignification.

3.1.4. Preparation of the Substrate for NFC Production via Ozonation. Using the optimized conditions (35\% pulp consistency, $\mathrm{pH}=2.5$, and ozone dosage of $0.87 \%$ ), ozonation of the KBP was performed. After the ozonation, the kappa

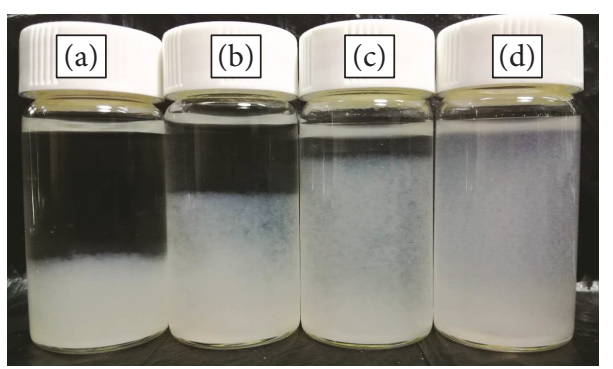

FIgURE 5: Appearance of the NFC product after standing still for $24 \mathrm{~h}$. Samples before (a) and after 1 (b), 3 (c), and 5 (d) passes through the microfluidizer.

number and the viscosity of the KBP decreased by $74.1 \%$ and $74.8 \%$, respectively, while the crystallinity and carboxylate content increased by $32.6 \%$ and $35.5 \%$, respectively (Table 3). The size of the BKP fibers was not dramatically changed by the ozonation treatment and had an average diameter of $10 \mu \mathrm{m}$ (Figures 4(a) and 4(b)). However, the distinct microfiber structure on the surface of the BKP fibers became less obvious due to the formation of fibrils and fragments.

3.2. Defibrillation of the KBP and the NFC Morphology. The treated KBP was then homogenized for NFC production. The treated KBP could smoothly pass through the microfluidizer, and a suspension of the NFC product was stably suspended after 5 cycles (Figure 5). In contrast, the untreated KBP seriously clogged the nozzles of the microfluidizer, which caused the NFC preparation to fail. The prepared NFC from the visible suspension from the 5-cycle homogenization was observed using TEM (Figures 6(a) and 6(b)). The TEM images of the isolated NFC showed widths of $10-20 \mathrm{~nm}$ and lengths up to $250 \mathrm{~nm}$. The high aspect ratio of the prepared NFC is consistent with the literature [25].

Ozone is an oxidizing agent with high oxidation potential. The ozonation treatment of KBP promoted fibrillation, which was advantageous for the preparation of NFC. This improved fibrillation can be explained by the decreased lignin composition and increased negative surface charge on the fibers. The ozone-induced delignification occurred via an electrophilic substitution reaction on the lignin units or 


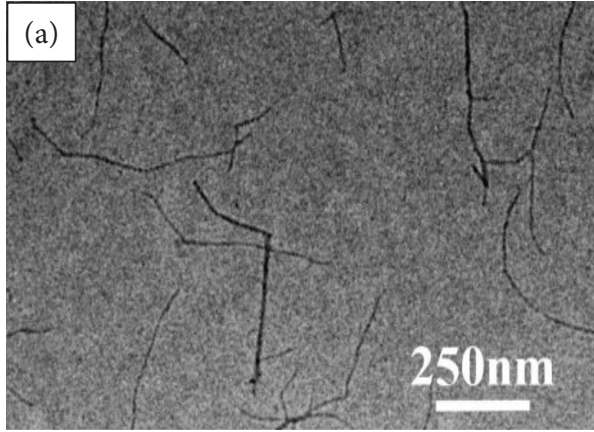

(a)

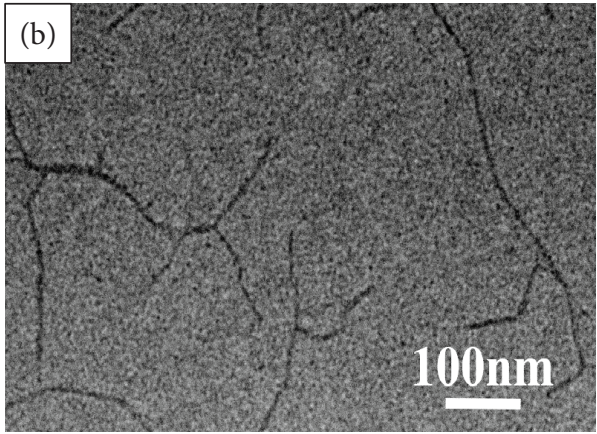

(b)

Figure 6: Appearance of the NFC with the scale bar of $250 \mathrm{~nm}$ (a) and $100 \mathrm{~nm}$ (b).

side chains $[26,27]$. Free radical reactions induced by the decomposition of ozone and lignin self-ozonation could also contribute to the improved delignification. Ozone could also enhance the hydrophilicity of the fibers, which may prevent the reformation of hydrogen bonds during mechanical defibrillation. In this study, ozone treatment of pulp had a similar effect as acid hydrolysis or TEMPO-mediated oxidation; however, the ozonation method is essentially free of environmental pollutants. The combination of ozone oxidation and high-pressure homogenization is an effective way to prepare nanocellulose.

\section{Conclusion}

NFC was successfully prepared from unbleached KBP using the combination of ozonation and high-pressure homogenization. The pulp consistency, $\mathrm{pH}$ value, and ozone dosage are important parameters in the ozonation of KBP. The ozonation treatment enabled the fibrillation of the KBP for the efficient preparation of NFC. The obtained NFC nanoparticles had a high aspect ratio.

\section{Data Availability}

The authors declare that all the supporting data for the results have been included in the submission.

\section{Conflicts of Interest}

The authors declare that they have no conflicts of interest.

\section{Acknowledgments}

The authors acknowledge the financial support of the National Science Foundation of China (31741023); Natural Science Foundation of Guangdong Province, China (2017A030310078); and Fundamental Research Funds for the Central Universities (D2172840) as well as the Opening Project of the Guangxi Key Laboratory of Clean Pulp \& Papermaking and Pollution Control (KF201603).

\section{References}

[1] K. L. Tovar-Carrillo, M. Tagaya, and T. Kobayashi, "Biohydrogels interpenetrated with hydroxyethyl cellulose and wooden pulp for biocompatible materials," Industrial \& Engineering Chemistry Research, vol. 53, no. 12, pp. 4650-4659, 2014.

[2] A. H. Basta, H. El-Saied, and V. F. Lotfy, "Performance of rice straw-based composites using environmentally friendly polyalcoholic polymers-based adhesive system," Pigment \& Resin Technology, vol. 42, no. 1, pp. 24-33, 2013.

[3] J. Pang, X. Liu, X. Zhang, Y. Wu, and R. Sun, "Fabrication of cellulose film with enhanced mechanical properties in ionic liquid 1-allyl-3-methylimidaxolium chloride (AmimCl)," Materials, vol. 6, no. 4, pp. 1270-1284, 2013.

[4] S. K. Rahimi and J. U. Otaigbe, "Polyamide 6 nanocomposites incorporating cellulose nanocrystals prepared by in situ ringopening polymerization: viscoelasticity, creep behavior, and melt rheological properties," Polymer Engineering and Science, vol. 56, no. 9, pp. 1045-1060, 2016.

[5] F. W. Herrick, R. L. Casebier, J. K. Hamilton, and K. R. Sandberg, "Microfibrillated cellulose: morphology and accessibility," Journal of Applied Polymer Science: Applied Polymer Symposium, vol. 37, pp. 797-813, 1983.

[6] A. F. Turbak, F. W. Snyder, and K. R. Sanberg, "Microfibrillated cellulose, a new cellulose product: properties, uses and commercial potential," Journal of Applied Polymer Science: Applied Polymer Symposium, vol. 37, pp. 815-827, 1983.

[7] I. Fatah, H. Khalil, M. Hossain et al., "Exploration of a chemo-mechanical technique for the isolation of nanofibrillated cellulosic fiber from oil palm empty fruit bunch as a reinforcing agent in composites materials," Polymer, vol. 6, no. 10, pp. 2611-2624, 2014.

[8] I. Besbes, S. Alila, and S. Boufi, "Nanofibrillated cellulose from TEMPO-oxidized eucalyptus fibres: effect of the carboxyl content," Carbohydrate Polymers, vol. 84, no. 3, pp. 975-983, 2011.

[9] W. Bai, J. Holbery, and K. Li, "A technique for production of nanocrystalline cellulose with a narrow size distribution," Cellulose, vol. 16, no. 3, pp. 455-465, 2009.

[10] P. Podsiadlo, S. Y. Choi, B. Shim, J. Lee, M. Cuddihy, and N. A. Kotov, "Molecularly engineered nanocomposites: layer-bylayer assembly of cellulose nanocrystals," Biomacromolecules, vol. 6, no. 6, pp. 2914-2918, 2005.

[11] K. Kato, V. N. Vasilets, M. N. Fursa, M. Meguro, Y. Ikada, and K. Nakamae, "Surface oxidation of cellulose fibers by vacuum 
ultraviolet irradiation," Journal of Polymer Science Part A: Polymer Chemistry, vol. 37, no. 3, pp. 357-361, 1999.

[12] S. Lemeune, H. Jameel, H. M. Chang, and J. F. Kadla, "Effects of ozone and chlorine dioxide on the chemical properties of cellulose fibers," Journal of Applied Polymer Science, vol. 93, no. 3, pp. 1219-1223, 2004.

[13] I. B. Tabar, X. Zhang, J. P. Youngblood, and N. S. Mosier, "Production of cellulose nanofibers using phenolic enhanced surface oxidation," Carbohydrate Polymers, vol. 174, pp. 120-127, 2017.

[14] J. Zhang, H. Song, L. Lin, J. Zhuang, C. Pang, and S. Liu, "Microfibrillated cellulose from bamboo pulp and its properties," Biomass \& Bioenergy, vol. 39, no. 8, pp. 78-83, 2012.

[15] N. Siddiqui, R. H. Mills, D. J. Gardner et al., "Production and characterization of cellulose nanofibers from wood pulp," Journal of Science and Technology, vol. 25, no. 6-7, pp. 709721, 2011.

[16] T. He, M. Y. Liu, and X. Tian, "Kinetics of ozone bleaching of eucalyptus kraft pulp and factors affecting the properties of the bleached pulp," BioResources, vol. 13, no. 1, pp. 425-436, 2018.

[17] L. Segal, J. J. Creely, A. E. Martin Jr, and C. M. Conrad, “An empirical method for estimating the degree of crystallinity of native cellulose using the X-ray diffractometer," Textile Research Journal, vol. 29, no. 10, pp. 786-794, 1959.

[18] F. Bettaieb, O. Nechyporchuk, R. Khiari, M. F. Mhenni, A. Dufresne, and M. N. Belgacem, "Effect of the oxidation treatment on the production of cellulose nanofiber suspensions from Posidonia oceanica: the rheological aspect," Carbohydrate Polymers, vol. 134, pp. 664-672, 2015.

[19] A. Ämmälä, H. Liimatainen, C. Burmeister, and J. Niinimäki, "Effect of tempo and periodate-chlorite oxidized nanofibrils on ground calcium carbonate flocculation and retention in sheet forming and on the physical properties of sheets," Cellulose, vol. 20, no. 5, pp. 2451-2460, 2013.

[20] T. Saito, M. Hirota, N. Tamura et al., "Individualization of nano-sized plant cellulose fibrils by direct surface carboxylation using TEMPO catalyst under neutral conditions," Biomacromolecules, vol. 10, no. 7, pp. 1992-1996, 2009.

[21] N. Yaqoob, K. Stack, and K. L. Nguyen, "TCF bleaching of eucalypt kraft pulp with oxone," Appita Journal, vol. 63, no. 5, pp. 381-386, 2010.

[22] M. B. Roncero, M. A. Queral, J. F. Colom, and T. Vidal, "Why acid $\mathrm{pH}$ increases the selectivity of the ozone bleaching processes," Ozone Science \& Engineering, vol. 25, no. 6, pp. 523534, 2003.

[23] S. Perincek, M. İ. Bahtiyari, A. E. Körlü, and K. Duran, "Ozone bleaching of jute fabrics," AATCC Review, vol. 7, no. 3, pp. 34$39,2007$.

[24] A. Seisto, K. Poppius-Levlin, and A. Fuhrmann, "Effect of ozone bleaching on the fibre properties of pine and birch kraft pulp," Cellulosic Pulps, Fibres and Materials, pp. 137-147, 2000.

[25] J. Xu, L. Tan, S. A. Baig, D. Wu, X. Lv, and X. Xu, "Dechlorination of 2,4-dichlorophenol by nanoscale magnetic $\mathrm{Pd} / \mathrm{Fe}$ particles: effects of $\mathrm{pH}$, temperature, common dissolved ions and humic acid," Chemical Engineering Journal, vol. 231, no. 17, pp. 26-35, 2013.
[26] E. Gilli, F. Schmied, S. Diebald, A. T. Horvath, C. Teichert, and R. Schennach, "Analysis of lignin precipitates on ozone treated kraft pulp by FTIR and AFM," Cellulose, vol. 19, no. 1, pp. 249-256, 2012.

[27] A. N. Mitrofanova, A. G. Khudoshin, and V. V. Lunin, "The ozonization of model lignin compounds in aqueous solutions catalyzed by $\mathrm{Mn}$ (II) ions," Russian Journal of Physical Chemistry A, vol. 84, no. 7, pp. 1141-1146, 2010. 


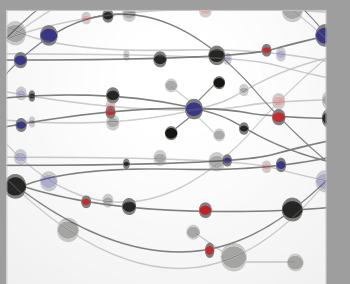

The Scientific World Journal
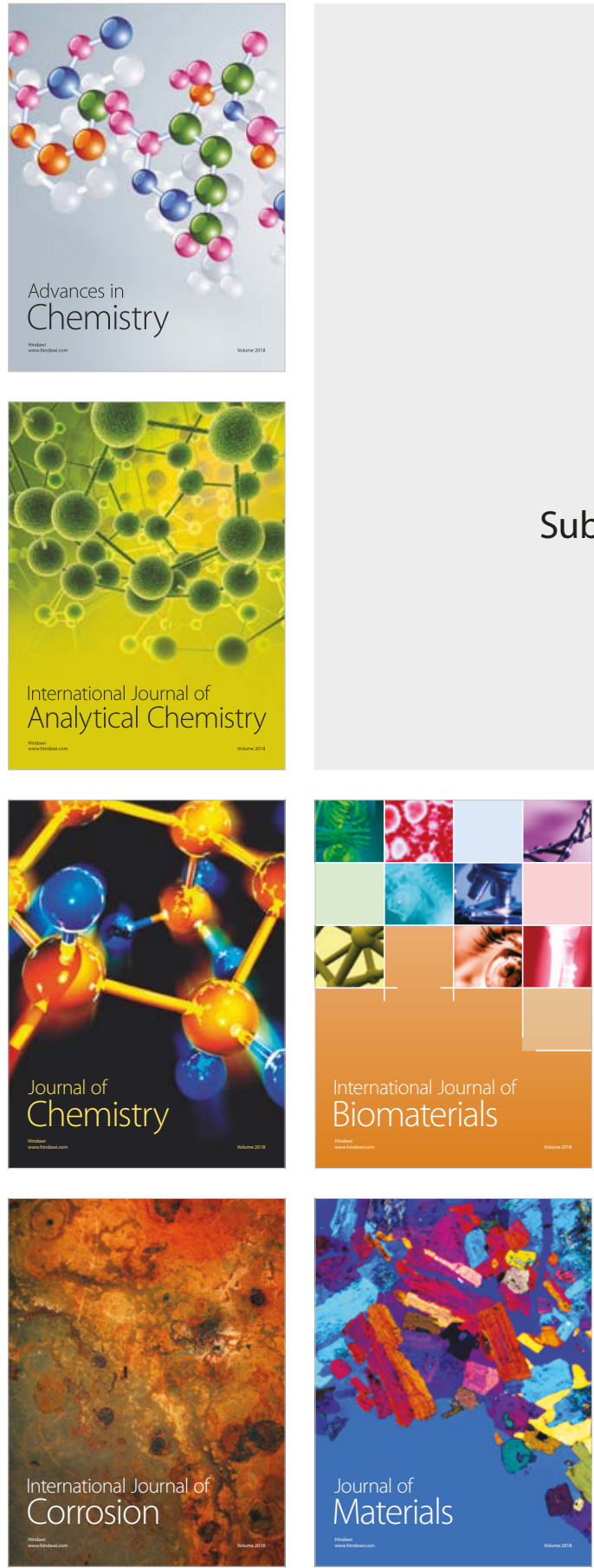

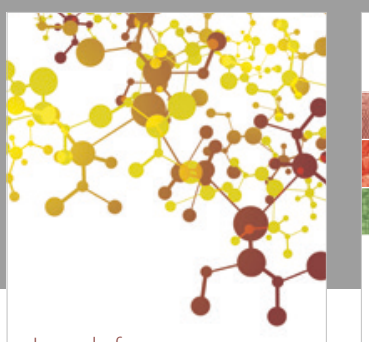

Journal of

Applied Chemistry
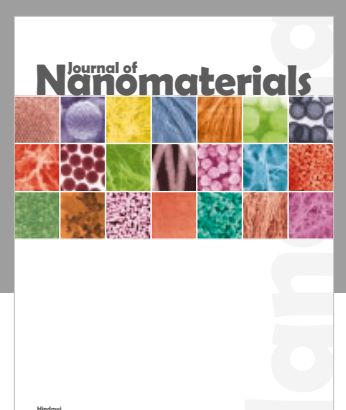

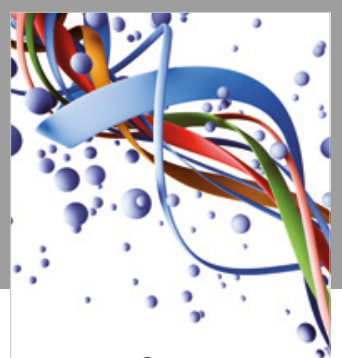

Scientifica

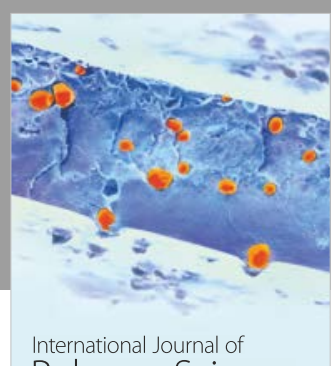

Polymer Science

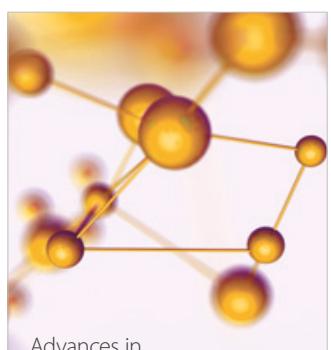

Physical Chemistry
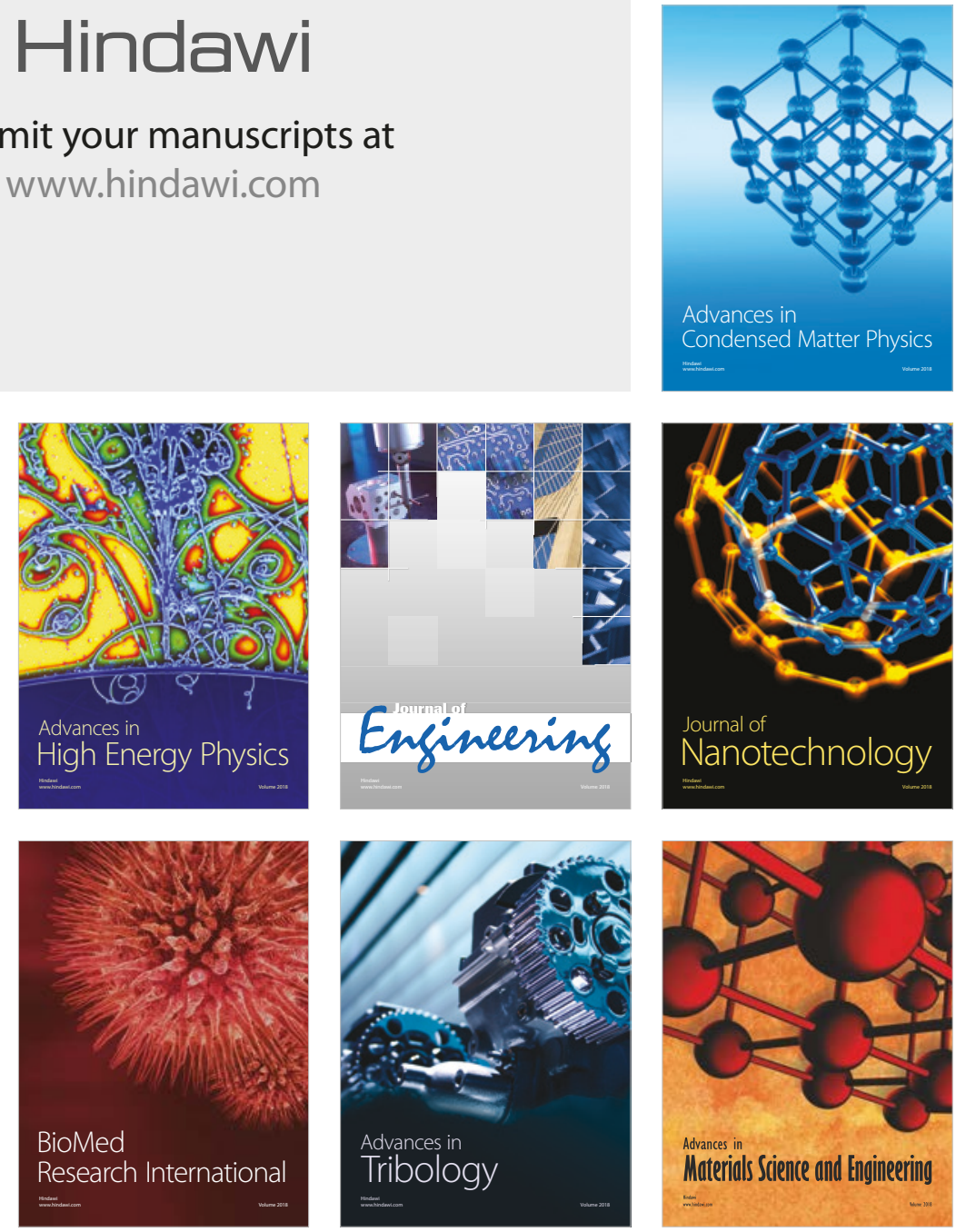